\title{
'Body-in-the-Loop' Optimization of Assistive Robotic Devices: A Validation Study
}

\author{
Jeffrey R Koller, Deanna H Gates, Daniel P Ferris, and C David Remy \\ University of Michigan, Ann Arbor, MI, USA \\ jrkoller@umich.edu
}

\begin{abstract}
Physiological measures, such as pain, anxiety, effort, or energy consumption, play a crucial role in the evaluation and development of assistive robotic devices. Physiological data are collected and analyzed by researchers and clinicians, and are often used to inform an iterative tuning process of a device and its controller. Currently, these data are collected then analyzed offline such that they are only evaluated after the experiment has ended. This makes any iterative design process tedious and time consuming since tuning must be done on a subject-by-subject basis and for a variety of tasks that the device is intended to be used for. To overcome these drawbacks, we are proposing a new type of human-machine interaction that is based on measuring and using physiological measurements in real-time. By continuously monitoring a physiological objective through a set of suitable sensors, we propose conducting an optimization of a set of controller parameters that shape the assistance provided by the device. In other words, we pose an optimization that includes the human body in the loop. This Body-in-the-Loop optimization allows for optimal subject specific control and has the potential to be used for controller adaptation to changing environments. We validated this concept in an extensive human subject study where we autonomously optimized the actuation onset of a pair of bilateral ankle exoskeletons to minimize user's metabolic effort.
\end{abstract}

\section{INTRODUCTION}

The control of a typical assistive robotic device relies on mechanically intrinsic measurements. These are measurements that come from the device itself and they are used to estimate the state of the human-machine system. These state estimates allow for the controller to determine the user's intent and the current phase of a given task. With this information, the controller allows the assistive device to aid the user in a variety of scenarios. Examples of mechanically intrinsic measurements include position, velocity, force, and impedance $[2,6,22]$. For example, in an upper extremity exoskeleton, force measurements between the user and the device can be used to predict the user's intended reaching trajectory [30]. Or in a lower limb prosthesis, acceleration measurements of the device can be used to estimate what phase of the gait cycle the user is currently in. This same acceleration measurement can determine if the user is intending to climb stairs or walk on level ground [26].

While mechanically intrinsic measurements estimate the state of the human-machine system, physiological measurements such as pain, anxiety, effort, or energy consumption, are commonly used to evaluate the performance of the device. Conclusions based on these physiological measurements are

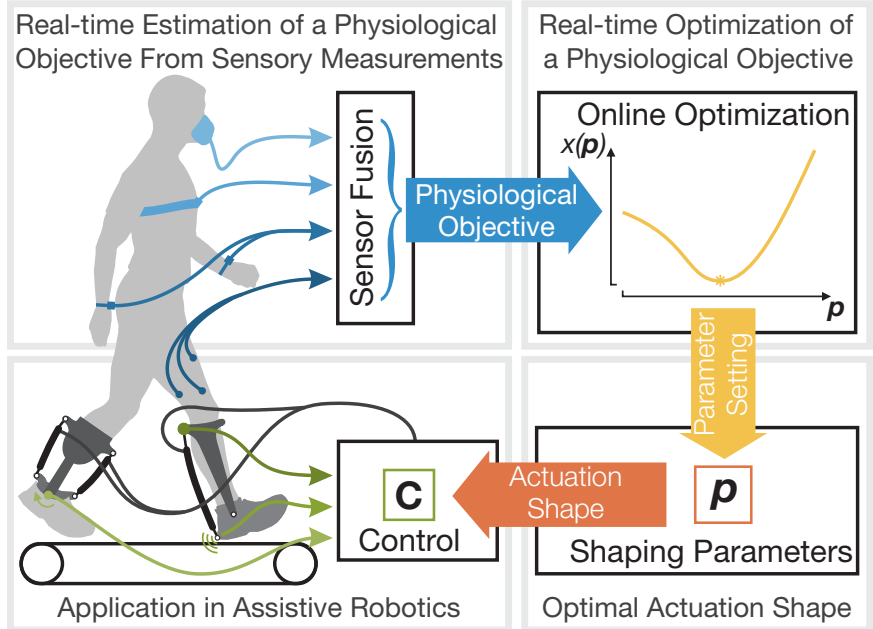

Fig. 1. Here is an illustration of the proposed concept of Body-in-the-Loop optimization. While a user is wearing an assistive robotic device (shown are devices that aid locomotion), a number of sensor readings are fused to form an estimate of a physiological measure $x$ (such as pain, anxiety, effort, or energy consumption). This physiological measure is used as the objective function in an online optimization that identifies optimal controller parameters $\boldsymbol{p}$. These targeted controller parameters influence the shape of assistance that is provided by the assistive device to the user. Additional intrinsic measurements from the device are used to estimate user intent and phasing of the assistance to drive the output of the controller $C$. Optimization is done with a human body in the loop which is a radically new way of human-machine interfacing.

then used to alter the design and to modify the shape of actuation to better assist the user. The process of using physiological measurements to shape actuation is an iterative, trial-and-error process. The measurements need to be taken during human subject testing and then analyzed by researchers or clinicians offline after testing. The results from this analysis are then used to drive future designs of actuation shape or tuning for a given device. This process is repeated until the desired results are achieved. For example, in an upper extremity prosthesis, measurements of user pain can be used to iteratively shape actuation such that users are most comfortable [32]. Or in a lower limb exoskeleton, measurements of energy consumption can be used to tune parameters that shape actuation profiles in an attempt to identify parameters that result in the largest reduction in the user's metabolic effort [31].

Clearly, both mechanically intrinsic measurements and physiological measurements are needed for the design and control of assistive robotic devices. However, in the current field of assistive robotic devices there is a divide between these measurements as they are not used concurrently in real- 


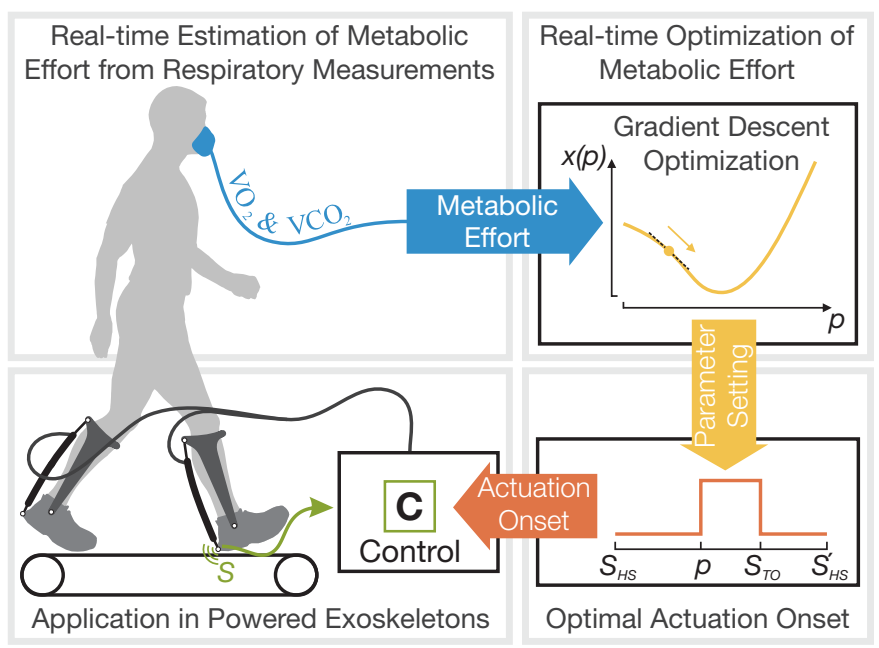

Fig. 2. In a validation study, we optimized the actuation onset (as parameter $p$ ) of bilateral ankle exoskeletons to minimize user's metabolic effort (as physiological objective $x(p)$ ). The exoskeleton controller used normalized stride time between consecutive heel strikes $\left(S_{H S}\right.$ and $\left.S_{H S}^{\prime}\right)$ as the state estimate to drive actuation. Actuation was turned on when the normalized phase of the stride $S$ was greater than the parameter setting $p$ and off when toe off $\left(S_{T O}\right)$ was detected. We implemented gradient descent optimization techniques to autonomously move toward a setting of $p$ that minimized $x(p)$ With this robotic device, we validated the Body-in-the-Loop techniques with eight healthy subjects, using a brute force mapping of subject specific cost landscapes as a ground truth for comparison.

time control. We are proposing a new method of humanmachine interfacing, one in which control parameters that shape assistance, $\boldsymbol{p}$, are optimally tuned and adjusted in realtime to minimize a physiological objective function from the user, $x(\boldsymbol{p})$. By including physiological measurements, $\mathbf{y}$, in the control scheme to estimate $x(\boldsymbol{p})$ and drive optimization, we are bringing the human body into the control loop. This Body-in-the-Loop approach has the potential to open up a new realm of assistive robotic control.

By including quantitative physiological feedback in a realtime optimization of shaping parameters, we can create robotic devices that learn and adapt in order to continuously provide optimal subject-specific assistance. The optimization process can be performed during the development of a controller for a new assistive system, as well as every time a particular device is fitted to a user. This allows adaptation to unique attributes such as the user's size, weight, or preferred movements, and it will greatly improve and accelerate the tedious task of controller tuning. It will substantially enhance the performance of current assistive robotic devices by optimally utilizing potential synergies between user and machine. In the long run, the proposed automated tuning can be applied not only when a device is built or fitted, but on a continuous basis as the person uses it. For example, in the case of a lower limb prosthesis, the controller could adapt in real-time to the type of shoes that the person is wearing, the terrain that is being traversed, or additional loads that a user might be carrying.

Felt et al. proved such an optimization was possible by optimizing step frequencies during normal walking of healthy subjects as a proof of concept [17]; however, this type of optimization has never been demonstrated on an actual assistive device until now. Here we expand upon Felt's work by presenting the first ever example of Body-in-the-Loop optimization driven by objective physiological measurements to optimize the control of an assistive robotic device. We build upon Felt's algorithm by including an oscillatory perturbation pattern, a growing sample window, and statistical confidence checks of each gradient estimate. All of these additions were crucial for improving the performance of Body-in-the-Loop optimization when applying these techniques to assistive devices due to the cost landscape being much shallower compared to that of Felt's step frequency study.

In this paper we have demonstrated the utility of Body-inthe-Loop techniques for the optimization of bilateral pneumatically actuated ankle exoskeletons during level ground walking. These exoskeletons provided additional power to aid in pushoff, reducing the metabolic effort required of the individual. We chose such a platform because devices targeting assistance at the ankle are commonly used in both a research and clinical setting due to the ankle's large contribution toward positive power generation during gait $[3,16,25,31]$. Our designed exoskeletons were simply controlled by a single shaping parameter, $p$, which controlled the actuation onset of the device. $p$ represents a normalized stride time threshold such that once a user's phase of normalized stride surpassed $p$, action was turned on. This is visualized in Figure 4 and has been a common control scheme used for basic science research with robotic ankle exoskeletons [11, 31]. The Body-in-theLoop optimization scheme tuned $p$ using metabolic effort as the physiological objective. We were able to estimate the user's metabolic effort in real-time via respiratory measurements using a mask as shown in Figure 3. Through the results from a extensive human subject study, we have shown that the proposed Body-in-the-Loop optimization techniques can autonomously move towards a metabolic minimum validating that Body-in-the-Loop optimization has the potential to be an impactful tool for future human-machine interactions.

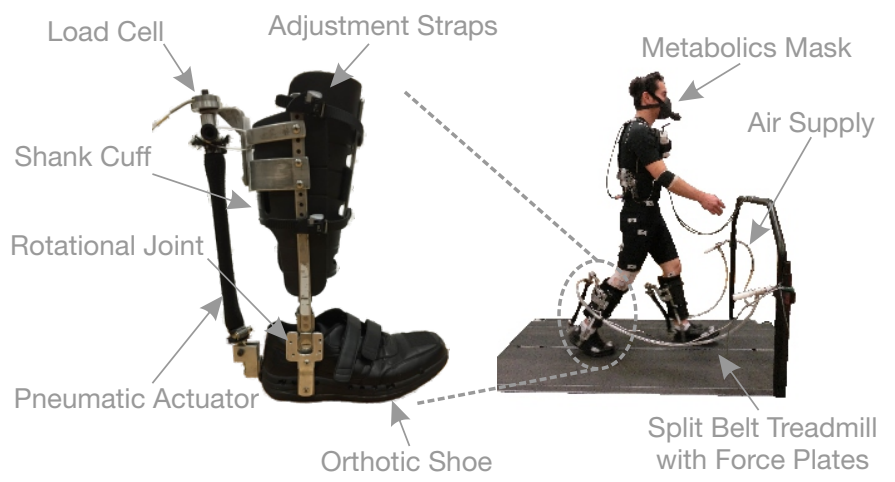

Fig. 3. The ankle exoskeletons used in this study were pneumatically actuated with custom built artificial pneumatic muscles attached posteriorly to assist with push off. During testing, subjects walked on a split belt treadmill with incorporated force plates to register heel strike and toe off events. Respiratory measurements were recorded in real time with a metabolics mask. The air pressure in the actuators was regulated with off board proportional pressure control valves (not shown). Adjustment straps allow fitting the device to different users. 


\section{A Pneumatically Actuated Robotic Ankle EXOSKELETON}

We used bilateral, single degree of freedom, pneumatically actuated ankle exoskeletons as a platform for testing the proposed Body-in-the-Loop optimization. We designed these exoskeletons based on previous work in the field [18].

\section{A. Hardware}

Each exoskeleton consisted of a shank and a foot component connected by a rotational joint. The shank diameter was adjustable via ratcheting straps to fit a variety of subject sizes. The shoes were customized orthotic shoes that had been outfitted with a stainless steel plate in the heel. This plate served to stiffen the heel of the shoe and provide attachment points for the actuators and rotational joints.

We actuated each exoskeleton with a custom made artificial pneumatic muscle [13] that had attachment points at the toprear of the shank and at the back of the heel. The moment arm between the rotational joint and the actuation attachment point at the heel was $10.1 \mathrm{~cm}$. The artificial muscle was connected to a proportional pressure control valve (MAC Valves, Wixom, MI) with a mechanical quick release valve (Parker Legris, Mesa, AZ) connected in series. Additionally, we attached a load cell (Omega Engineering, Stamford, Connecticut) in series with the artificial muscle to record actuation kinetics. This actuation configuration had an electromechanical delay of $28 \mathrm{~ms}$. In optimal controller configurations, this exoskeleton can provide approximately $50 \%$ of the torque required at the ankle during normal walking. Each exoskeleton, artificial

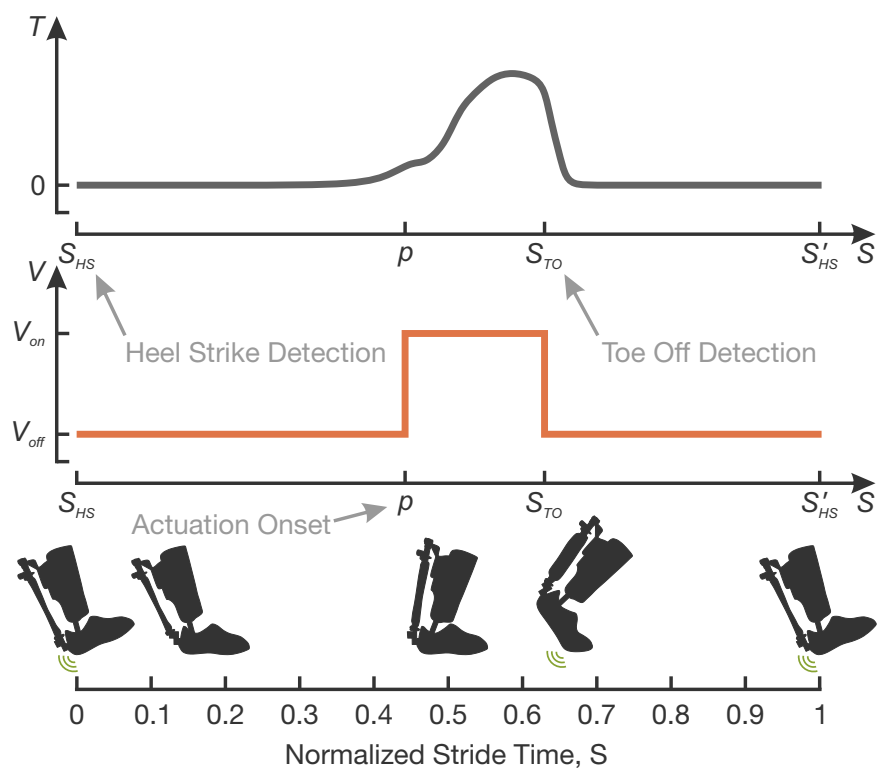

Fig. 4. The control signal that was sent to the pressure control valves was based upon normalized stride time, $S$. The bottom graph shows a single stride of the exoskeleton where $S_{H S}=0, S_{T O} \approx 0.63$, and $S_{H S}^{\prime}=1$. The middle graph shows how actuation is turned on once $S \geq p$ and off once $S \geq S_{T O}$. The top graph shows an example of the expected torque output of the exoskeleton. This profile will change dependent upon what $p$ is set to and as a function of user specific ankle kinematics during walking. pneumatic muscle, and load cell had a combined weight of $2.02 \mathrm{~kg}$. This weight was slightly larger than other state of the art exoskeletons in the field, yet it was within the weight range of exoskeletons that have previously shown significant reductions in metabolic effort $(0.67-2.30 \mathrm{~kg}[31,33])$.

\section{B. Control}

We controlled the exoskeleton using a simple state-based control strategy that has been presented in previous ankle exoskeleton work [11, 31]. The controller was simply an 'onoff' control scheme that was triggered by a single state, $S$, crossing a threshold value, $p . p$ is the shaping parameter that we optimized for during validation testing. $S$ was a measure of normalized stride time where $S=0$ and $S=1$ corresponded to consecutive heel strikes of the same leg. We used vertical ground reaction forces on the treadmill to detect heel strike, $S_{H S}$, and toe off, $S_{T O}$, of each stride. We calculated stride times from the time between consecutive heel strikes of the same leg and then normalized them by an average stride time to calculate $S$. We calculated the average stride time using a finite impulse response filter with a tap size of five.

When $p \leq S \leq S_{T O}$, actuation was turned on. Actuation was turned off otherwise. When the controller was in the on state, we pressurized the artificial pneumatic muscles with 600 $\mathrm{kPa}$ of pressure and when in the off state with $150 \mathrm{kPa}$ of pressure. The reason for a non-zero off state pressure was to deplete any dead space in the tubing and muscle. This allowed for a quicker actuation response and limited electromechanical delay in our control system. The off pressure of $150 \mathrm{kPa}$ did not result in any inflation of the artificial pneumatic muscle and thus no tension was registered by the load cells during the off state due to actuation pressure.

\section{Metabolic Effort as a Physiological Cost FUNCTION}

For this study we used metabolic effort as the physiological cost function, $x(p)$, to minimize. In relation to metabolic effort, $x(p)$ is also known as the instantaneous energetic cost [34]. Metabolic effort is a measure of how much energy the human body is expending during any task and can be estimated from a variety of physiological measurements such as oxygen consumption, heart rate, oxygen saturation in the blood, or muscle activity $[8,20,24,27]$. Most commonly, metabolic effort is estimated from oxygen consumption via respiratory measurements, $\mathbf{y}$, and is a primary method for evaluation of assistive robotic devices [15]. In collecting respiratory measurements, subjects wear a mask that samples the oxygen and carbon dioxide content of each individual breath. Using equations from Brockway [8] and normalizing by subject mass, we convert these respiratory measurements $\left(\frac{m L}{s e c}\right)$ to measurements of energy consumption $\left(\frac{W}{k g}\right)$.

Although respiratory measurements give us a fairly accurate estimate of metabolic effort, there are significant sensor dynamics that prevent measuring $x(p)$ directly. Respiratory measurements are ideally meant for long testing trials at steady state conditions due to a long delay between the instantaneous 
energetic demand and the physical respiratory measurements. When muscles need instantaneous energy they pull from local energy storage in the form of adenosine triphosphate (ATP). These local storages of ATP are then replenished through a number of processes that require oxygen pulled from the blood stream. It takes time for this oxygen depleted blood to travel from the muscle location to the lungs, so there is a delay between this initial recruitment of energy and the observation of increased oxygen consumption via respiratory measurements. Because of this delay, the dynamics of the human metabolic system are commonly modeled as a first order system, having a time constant $\tau$ of approximately 40 seconds during walking $[29,34,35]$. It is common practice to have subjects walk at a given condition for at least three minutes to allow for metabolic effort to stabilize prior to collecting measurements for analysis due to this large time constant. Additionally, respiratory measurements are very sparse because only one measurement is made per breath. During normal walking subjects take approximately 19 breaths per minute which results in a sample rate of $0.32 \mathrm{~Hz}$ [7]. On top of all that, respiratory measurements are incredibly noisy, having a signal to noise ratio of approximately four [29, 35]. Given this sparse sample rate and high noise level, it is typical to average respiratory measurements across multiple minutes to achieve a single data point estimate of $x(p)$.

Dynamic delay, sparse sampling, and large inter-breath variability all make for using respiratory measurements to drive an online optimization increasingly difficult. In order

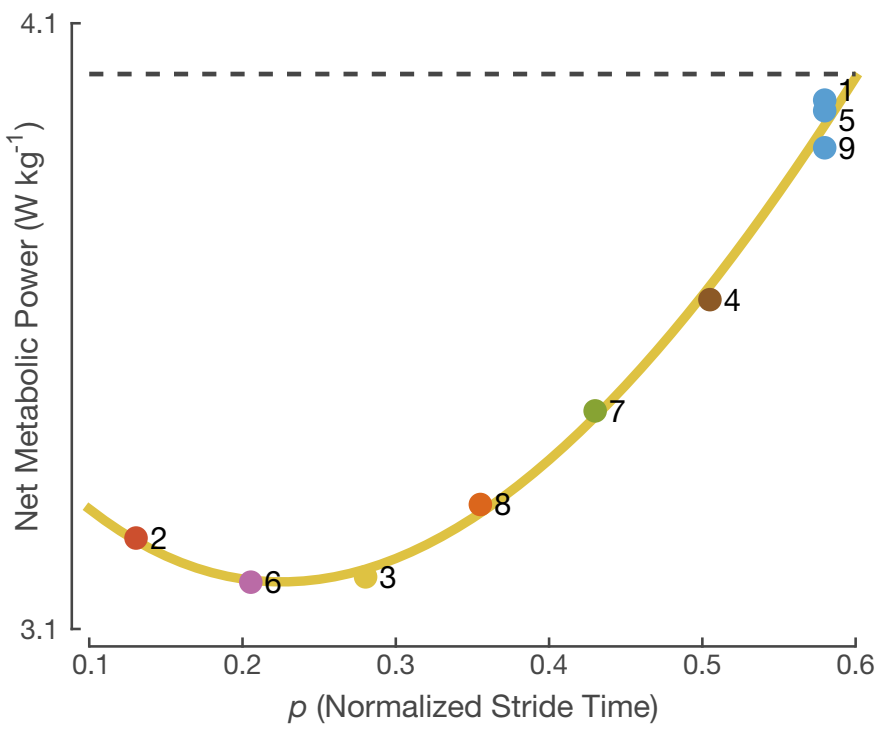

Fig. 5. To provide a ground truth for comparison, the cost landscape for each individual subject was found by averaging the final three minutes of metabolic data from each powered condition during a steady state cost mapping trial. Each average (shown here for a pilot subject) is represented by a colored dot and numbered according to the testing sequence. The subject's standing metabolic power has been subtracted from all shown values. A third order polynomial was fit to these steady state values (shown in yellow). For comparison, the dashed line shows the subject's metabolic power when walking in the unpowered exoskeletons. The highest parameter setting was repeated three times $(1,5,9)$ to test for learning and fatigue effects. to use this information in a real-time optimization, we need to establish a suitable sensor model of respiratory measurements. The input to this sensor is the instantaneous energetic cost $x\left(p_{i}\right)$. The output are individual breath measurements of energy consumption $y_{i} . i$ is the breath number and replaces a notion of time in this discrete breath-by-breath process. Approximating the metabolic dynamics as a discrete linear first order system, we relate respiratory measurements, $y_{i}$, of each breath $i$, to metabolic effort, $x\left(p_{i}\right)$ :

$$
y_{i}=\left(1-\frac{h_{i}}{\tau}\right) y_{(i-1)}+\frac{h_{i}}{\tau} x\left(p_{i}\right) .
$$

In this equation, $h_{i}$ is the time since the previous breath and $\tau$ is the time constant of the metabolic system [17].

\section{Optimization of Metabolic EfFort}

Based on this sensor model, we developed an algorithm for the real-time optimization of metabolic effort. Please note that the figures in this section are representing exemplary data from a single pilot subject. Data from the complete validation study is presented in Section V-B.

\section{A. Establishing a Ground Truth}

In order to objectively evaluate the proposed Body-in-theLoop optimization techniques, we first established a ground truth of the cost landscape. To this end, we mapped subject's energetic cost landscapes using a steady state cost mapping (SSCM) protocol similar to Felt et al. [17]. This SSCM protocol is a type of parameter study common in assistive robotics to brute force map $x(p)[12,14,31]$.

The SSCM protocol that we implemented had subjects standing in place for four minutes to measure their standing metabolic effort. We then had subjects walk with the exoskeleton in an unpowered condition followed by nine powered conditions. Subjects walked for six minutes in each condition. This process was performed continuously where subjects were only given verbal warnings before starting the treadmill, powering on the exoskeletons, and powering off the exoskeletons. Subjects walked at seven different powered parameter settings $(p=0.13,0.21,0.28,0.36,0.43,0.51$, and 0.58 ) for nine different powered conditions. The first, fifth, and ninth powered conditions were always $p=0.58$. All intermediate powered conditions were randomized. The repeated $p=0.58$ allowed us to analyze learning effects and fatigue in subjects. If we saw significant differences in the first, fifth, and ninth parameter's steady state values, we could assume that the subject was still learning and adapting to the device, or that they were becoming fatigued. We chose the parameter of $p=0.58$ for this repetition, because this setting turns actuation on for the shortest amount of time. Since $S_{T O} \approx 0.63$ [31], the device was only powered for $\sim 60 \mathrm{~ms}$ at this setting. By starting and ending with this parameter setting, we were able to ease subjects in and out of powered conditions in the smoothest and safest way possible.

After data collection, we averaged the final three minutes of data from each powered condition in the SSCM data set 
to generate an estimate of the instantaneous energetic cost, $\bar{x}(p)$. In the realm of assistive devices, it is common practice to estimate $\bar{x}(p)$ with a third order polynomial fit [14, 23, 31]. This representation of $\bar{x}(p)$ versus $p$ is shown in Figure 5.

\section{B. Online Optimization}

Our proposed online optimization scheme is based upon gradient descent methods [4]. We allowed the algorithm to run for a set amount of time $\left(t_{\text {end }}\right)$ during which it was fully autonomous meaning there was no human intervention once the algorithm had begun. An overview of our algorithm is presented below as pseudo-code with the variables defined in the proceeding subsections.

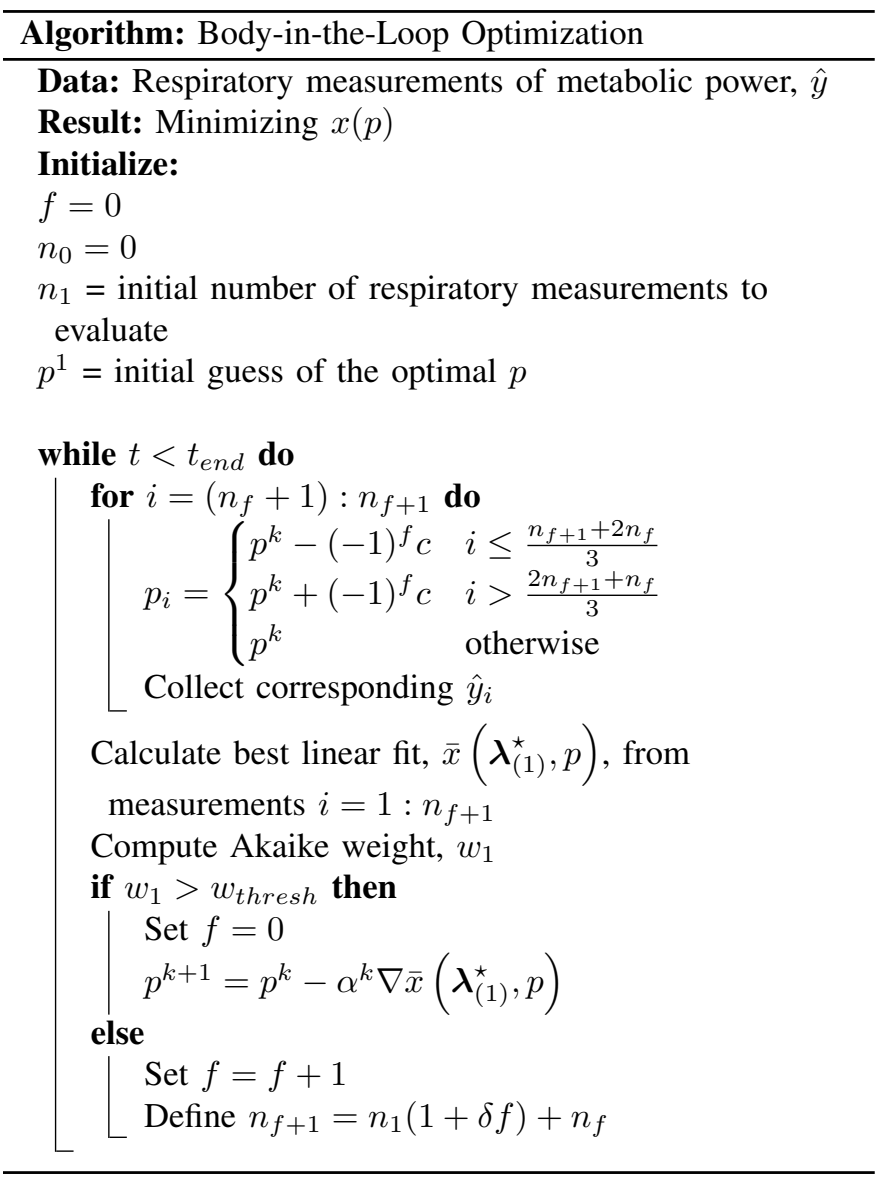

1) Parameter Exploration about $p^{k}$ : To obtain an estimate of the gradient of the physiological objective at $p^{k}$, we systematically explored parameter settings surrounding $p^{k}$. We did this by initially evaluating below, at, and above $p^{k}$ for a total of $n_{1}$ breaths. If we were not confident in the gradient estimate of $p^{k}$ after $n_{1}$ breaths (ie it failed a statistical test of confidence) more data was collected. In these additional evaluations we collected data about $p^{k}$ in the reverse order of the previous exploration for a total of $n_{1}(1+\delta f)$ more breaths. The variable $f$ counted the number of evaluations, and a larger number of breaths was collected in each subsequent evaluation. We reevaluated the gradient for all measurements collected about $p^{k}$ and repeated as necessary.
This oscillatory sampling pattern about $p^{k}$ was defined as follows such that $n_{f}$ and $n_{f+1}$ were breath numbers, $i$ was the breath count at $p^{k}, c$ was the perturbation size, and $f$ was the number of failed statistical tests at $p^{k}$.

$$
p_{i}= \begin{cases}p^{k}-(-1)^{f} c & i \leq \frac{n_{f+1}+2 n_{f}}{3} \\ p^{k}+(-1)^{f} c & i>\frac{2 n_{f+1}+n_{f}}{3} \\ p^{k} & \text { otherwise }\end{cases}
$$

We initialized $n_{0}, n_{1}, f$, and $p^{1}$ before the algorithm began. $n_{1}$ was a user defined input for the initial number of respiratory measurements to consider per oscillation and $p^{1}$ was an initial guess of the optimal $p$ location. If the gradient fit failed a statistical test of confidence then we incremented $f$ and calculated $n_{f+1}$ as

$$
n_{f+1}=n_{1}(1+\delta f)+n_{f}
$$

where $\delta$ represents a user defined growth factor.

2) Gradient Estimate: Based on the measurements $y_{i}$ at the known parameter settings $p_{i}$, we estimated a linear representation of $x(p)$ and extracted the gradient from this representation. This linear representation was defined by a set of coefficients $\boldsymbol{\lambda}$

$$
\bar{x}\left(\boldsymbol{\lambda}_{(1)}, p\right)=\lambda_{0}+\lambda_{1} p
$$

In this notation, the gradient of the linear function, $\nabla \bar{x}\left(\boldsymbol{\lambda}_{(1)}, p\right)$, is simply $\lambda_{1}$. By running this linear approximation through (1), we produce an estimate of metabolic effort $\bar{y}_{i}$ as a function of $\boldsymbol{\lambda}$ and $p_{i}$ :

$$
\bar{y}_{i}=\left(1-\frac{h_{i}}{\tau}\right) \bar{y}_{(i-1)}+\frac{h_{i}}{\tau} \bar{x}\left(\boldsymbol{\lambda}_{(1)}, p_{i}\right) .
$$

Starting from an initial value $\bar{y}_{1}$, the series of estimated breath measurements $\bar{y}_{i}$ can be obtained by recursively evaluating (5). The resulting expression is linear with respect to a $\bar{y}_{1}$ and $\boldsymbol{\lambda}$ :

$$
\left(\begin{array}{c}
\bar{y}_{1} \\
\vdots \\
\bar{y}_{n}
\end{array}\right)=\mathbf{A}\left(\begin{array}{c}
\bar{y}_{1} \\
\lambda_{0} \\
\lambda_{1}
\end{array}\right)
$$

A is a $n \times 3$ matrix whose elements are calculated recursively based upon $\tau, h_{i}$, and $p_{i}$ [17].

$$
\mathbf{A}_{i, j}=\left\{\begin{array}{rl}
1 & i=1, j=1 \\
0 & i=1, j>1 \\
\mathbf{A}_{i-1, j}\left(1-\frac{h_{i}}{\tau}\right) & i>1, j=1 \\
\mathbf{A}_{i-1, j}\left(1-\frac{h_{i}}{\tau}\right)+\frac{h_{i}}{\tau} \frac{\partial \bar{x}\left(\boldsymbol{\lambda}_{(1)}, p_{i}\right)}{\partial \lambda_{j-2}} & i>1, j>1
\end{array}\right.
$$

For a given set of breath measurements $\hat{y}_{i}$, we employ the pseudo-inverse of $\mathbf{A}$ (denoted as $\mathbf{A}^{+}$) to solve for the optimal initial breath, $\bar{y}_{1}^{\star}$, and optimal linear fit, $\boldsymbol{\lambda}_{(1)}^{\star}$, that yield a leastsquare fit between $\hat{y}_{i}$ and $\bar{y}_{i}$ :

$$
\left(\begin{array}{c}
\bar{y}_{1}^{\star} \\
\lambda_{0}^{\star} \\
\lambda_{1}^{\star}
\end{array}\right)=\mathbf{A}^{+}\left(\begin{array}{c}
\hat{y}_{1} \\
\vdots \\
\hat{y}_{n}
\end{array}\right) \text {. }
$$


That is, we identified model coefficients that provide a best fit between the linear model $\bar{x}\left(\boldsymbol{\lambda}_{(1)}, p\right)$ and the true underlying relationship $x(p)$. Through this process, a new gradient $\nabla \bar{x}\left(\boldsymbol{\lambda}_{(1)}, p\right)$ was established every time a total of $n_{f+1}$ measurements had been taken about $p^{k}$.

In addition to fitting a linear model (as per (8)), we used the same process to simultaneously identify a purely constant (zeroth order) model, $\bar{x}\left(\lambda_{(0)}^{\star}, p\right)$ :

$$
\bar{x}\left(\lambda_{(0)}, p\right)=\lambda_{0} .
$$

We used this model to evaluate confidence in the gradient fit. In this model $\mathbf{A}$ was a $n \times 2$ matrix.

3) Confidence in the Gradient: Due to the poor signal to noise ratio of respiratory measurements, we implemented a statistical test to evaluate how confident we were in any given linear fit prior to acting upon it in the gradient descent algorithm. To this end, we compared the optimal first order fit to data about $p^{k}$ to the optimal zeroth order fit to the same data. If the first order fit was statistically better at describing the data than the zeroth order fit (that is, when a gradient fit the data better than a flat line) the gradient was used to update $p^{k}$. The implemented statistical test used Akaike weights and a user defined threshold to establish confidence in a gradient estimate. From simulations of the Body-in-the-Loop optimization, we found this method to be more conservative in nature compared to other statistical tests.

The Akaike Information Criterion, $\mathrm{AIC}_{l}$, is a measure used when comparing model $l$ to multiple other model fits and is meant to determine which model in a given set is the best fit

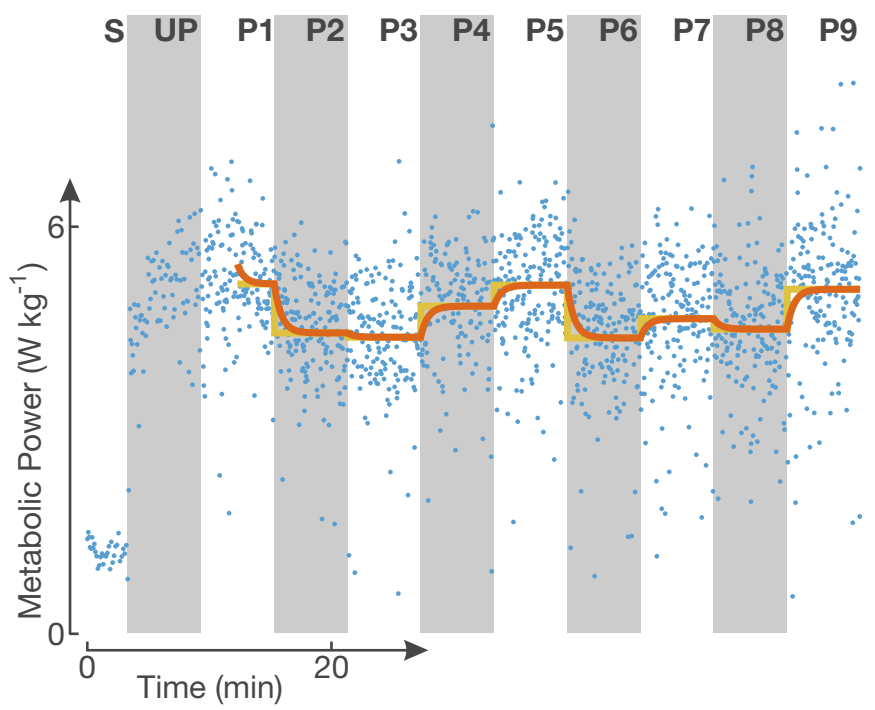

Fig. 6. We used the SSCM data to estimate subject specific $\tau$ 's. The raw metabolic data $\left(\hat{y}_{i}\right)$ is shown in blue, the instantaneous metabolic cost $\left(\bar{x}\left(p_{i}\right)\right)$ is shown in yellow, and the metabolic estimate $\left(\bar{y}_{i}\right)$ is shown in orange. During the SSCM we recorded standing metabolics from the subject for four minutes (S), unpowered walking in the device for six minutes (UP), and nine different powered conditions for six minutes each (P1-P9). The data shown here is from a pilot subject that had a metabolic time constant of 36.6 seconds. This graph also illustrates the sparse data and high noise levels that are typical for respiratory measurements.

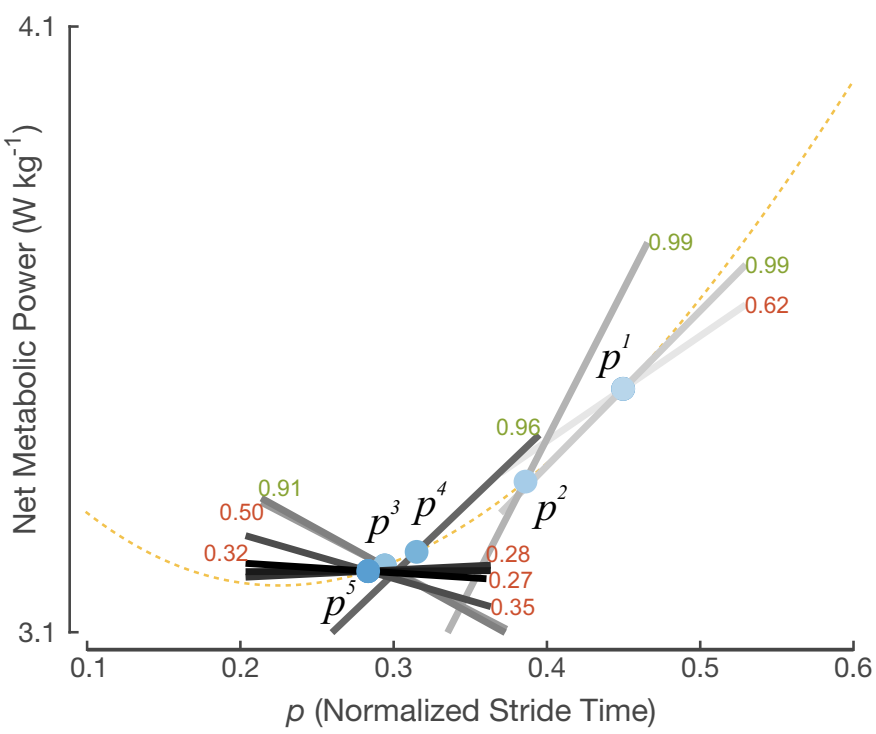

Fig. 7. Here is an example of a gradient based online optimization run that was started from $p=0.45$. The presented data is from the same pilot subject as the data in Figures 5 and 6. Gradient estimates darken to represent progress through the optimization routine. The gradients are overlaid on the ground truth for visualization purposes. $w_{1}$ values that correspond to the confidence in a gradient estimate are written touching their corresponding fits with green representing $w_{1}>w_{\text {thresh }}$ and red representing $w_{1} \leq w_{\text {thresh }}$. In this particular case the optimizer finished at $p=0.28$ where the minimum identified from the SSCM was $p=0.23$. According to the SSCM polynomial fit, this error resulted in an increase of $0.023 \mathrm{~W} \mathrm{~kg}^{-1}$ in metabolic power for this pilot subject.

$[1,9]$. In this framework, $\mathrm{AIC}_{l}$ can be calculated as

$$
\mathrm{AIC}_{l}=n_{f+1} \ln \left(\frac{e_{l}}{n_{f+1}}\right)+2(m+1) .
$$

where $e$ is the sum of squared error between $\bar{y}$ and $\hat{y}$ (defined in (14)), and $m$ is the number of elements of $\boldsymbol{\lambda}$. By traditional standards, the lowest $\mathrm{AIC}_{l}$ value from the set of models, min AIC, is the best model fit of the group; however, the min AIC value alone does not give a sense as to how much better the best fit truly is. We used Akaike weights, $w_{l}$, to give us a sense of confidence in the model fits. $w_{l}$ can range from 0 to 1 and can be interpreted as the probability that model $l$ is the best model within a group of $R$ total model fits $[9,10]$. $w_{l}$ is defined as

$$
w_{l}=\frac{\exp \left\{-0.5 \Delta_{l}\right\}}{\sum_{r=1}^{R} \exp \left\{-0.5 \Delta_{r}\right\}}
$$

where $\Delta_{l}=\mathrm{AIC}_{l}-\min \mathrm{AIC}$.

In the described optimization scheme we used the Akaike weights to compare a first order fit, $\bar{x}\left(\boldsymbol{\lambda}_{(1)}^{\star}, p\right)$, to a zeroth order fit, $\bar{x}\left(\lambda_{(0)}^{\star}, p\right)$, meaning $R=2$ in (11). Only if the Akaike weight associated with $\bar{x}\left(\boldsymbol{\lambda}_{(1)}^{\star}, p\right), w_{1}$, was greater than a user defined threshold, $w_{\text {thresh }}$, the gradient descent algorithm took a step. Otherwise, more data was collected at the current parameter setting $p^{k}$.

4) Updating $p^{k}$ : If $w_{1}>w_{\text {thresh }}$ then the evaluation parameter was updated as

$$
p^{k+1}=p^{k}-\alpha^{k} \nabla \bar{x}\left(\boldsymbol{\lambda}_{(1)}^{\star} \cdot p\right) .
$$


TABLE I

VARIABLES USED IN BODY-IN-THE-LOOP OPTIMIZATION

\begin{tabular}{|c|c|c|c|}
\hline Variable & Definition & Units & Value \\
\hline$t_{\text {end }}$ & Termination Time & Minutes & 50 \\
\hline$c$ & Perturbation Size & - & 0.08 \\
\hline$n_{1}$ & Initial Breath Evaluation Size & Breaths & 90 \\
\hline$p^{1}$ & Initial Evaluation Parameter & - & Randomized \\
\hline$\delta$ & Evaluation Growth Factor & - & 0.5 \\
\hline$w_{\text {thresh }}$ & Akaike Weight Threshold & - & 0.7 \\
\hline$A_{0}$ & Gain Scheduling Parameter & - & 1 \\
\hline$\alpha_{0}$ & Gain Scheduling Parameter & $\frac{1}{\mathrm{Kkg}^{-1}}$ & 500 \\
\hline$\gamma$ & Gain Scheduling Parameter & - & 0.75 \\
\hline
\end{tabular}

In this equation, $\alpha^{k}$ is a scheduling gain $[4,17]$ defined as

$$
\alpha^{k}=\frac{A_{0} \alpha_{0}}{A_{0}+k^{\gamma}} .
$$

where $\alpha_{0}, A_{0}$, and $\gamma$ are user defined constants with $\gamma=(0,1] . k$ was only incremented if a step was taken at which point $f$ was set to zero. If $w_{1} \leq w_{\text {thresh }}$, then no step was taken and $f$ was incremented while $k$ remained constant.

\section{Implementation}

The presented optimization algorithm was implemented in Python. The Python scripts took in measures of $\hat{y}_{i}$ and communicated $p_{i}$ values via serial connection to a real-time control board (dSPACE, Inc., Northville, MI) that ran the control loop described in Section II-B. This real-time control board also received measures of vertical ground reaction force from the instrumented treadmill and determined instances of heel strike or toe off. Based on these gait events, the controller calculated appropriate actuation control signals and sent them to the pressure valves. The valves regulated the pressurized artificial pneumatic muscles proportional to the received control signals. A list of parameter values used during actual data collection can be found in Table I. We chose these values based upon simulation results of the optimization routine. These simulations assumed a Gaussian distribution in respiratory measurement noise, an average metabolic time constant, and a hypothetical physiological objective function. For safety reasons, we bound $p^{k}$ between 0.21 and 0.50 in implementation, yet these limits were never needed to be enforced during actual subject testing.

An important parameter in the algorithm is the time constant $\tau$ of the metabolic dynamics. There are a number of ways to estimate $\tau$, and it was shown in prior work that it could simply be approximated by a constant value of 40 seconds [17]. For this work, we chose to use the SSCM data to estimate subject specific estimates of $\tau$ as this data was readily available. We averaged the final three minutes of each powered condition to establish a breath dependent estimate of the instantaneous metabolic cost, $\bar{x}\left(p_{i}\right)$. Using $\bar{x}\left(p_{i}\right)$ and (1), we estimated metabolic cost for a given $\tau$ and $\bar{y}_{1}$. Constraining $\tau$ and $\bar{y}_{1}$ to positive values, we used MATLAB's Optimization Toolbox (The MathWorks, Inc., Natick, MA) to determine the $\tau$ and $\bar{y}_{1}$ that resulted in the smallest sum of squared error between actual metabolic measurements, $\hat{y}_{i}$, and estimated metabolic measurements, $\bar{y}_{i}$. That is to say,

$$
\min _{\tau, \bar{y}_{1}} e=\sum_{i=1}^{n}\left(\hat{y}_{i}-\bar{y}_{i}\right)^{2} .
$$

We only used powered condition data for this fit beginning from the steady state of the first parameter as shown in Figure 6. An example of the optimization being performed with this subject specific $\tau$ and the variable values presented in Table I is shown in Figure 7.

\section{EXPERIMENTAL EVALUATION}

To validate the Body-in-the-Loop optimization techniques, we tested the presented algorithm on healthy subjects wearing the bilateral ankle exoskeletons described in Section II. All testing was in accordance to the University of Michigan's Medical School's Institutional Review Board (HUM00070022), and all subjects gave informed written consent to participate in the study prior to testing.

\section{A. Experimental Protocol}

We tested the proposed optimization techniques on nine healthy participants. Of these nine participants, four had never walked in a powered exoskeleton before. Only male participants were tested due to the large shoe size requirement of the exoskeleton design. One subject (Subject 2) was deemed an outlier due to inexplicably noisy respiratory measurements (a signal to noise ratio $\sim 2$ ). The eight remaining subjects presented here (age: $23 \pm 6$ years; body mass: $72.2 \pm 5.3 \mathrm{~kg}$; height: $179.4 \pm 7.7 \mathrm{~cm}$; mean $\pm \mathrm{SD}$ ) walked in bilateral robotic ankle exoskeletons during three separate testing days.

On the first two days of testing, we performed the SSCM protocol explained in Section IV-A to establish subject specific ground truths. Day one was used as a training day for subjects to learn and adapt to walking in the device. The data from day one has therefore been disregarded in this analysis. We used the SSCM data from the second day to establish a ground truth and to estimate subjects' time constants $\tau$. All differences in steady state metabolic measurements of the repeated first, fifth, and ninth parameters were within the expected levels of measurement noise. On the third day of testing, we tested the proposed optimization scheme on each subject starting from a randomized start position, $p^{1}$. The optimization algorithm was run for 50 minutes before terminating. All walking took place on a split belt treadmill (Bertec Corporation,Columbus, $\mathrm{OH}$ ) at $1.2 \mathrm{~ms}^{-1}$ and all measurements of metabolic effort were taken using a portable open-circuit indirect spirometry system (CareFusion Oxycon Mobile, Hoechberg, Germany).

TABLE II

Average Results

\begin{tabular}{|c|c|c|}
\hline & Mean & Standard Deviation \\
\hline $\begin{array}{c}\text { Time Constant } \tau \\
\text { (Seconds) }\end{array}$ & 47.8 & \pm 12.4 \\
\hline $\begin{array}{c}\text { Absolute Error in } \\
\text { Minimum } p \\
\text { (Unitless) }\end{array}$ & 0.097 & \pm 0.077 \\
\hline
\end{tabular}




\section{B. Results}

Table II shows the average results from all eight subjects. On average our optimization techniques terminated at a parameter setting 0.097 away from the optimal parameter setting identified from the SSCM protocol. According to the established subject specific ground truth curves, this parameter error would result in an average increase of $0.056 \mathrm{~W} \mathrm{~kg}^{-1}$ in metabolic power. This increase is well within the range of sensor noise for respiratory measurements. However, to put this increase of $0.056 \mathrm{~W} \mathrm{~kg}^{-1}$ into perspective, subjects experienced a $0.80 \pm 0.18 \mathrm{~W} \mathrm{~kg}^{-1}$ (mean $\left.\pm \mathrm{SD}\right)$ decrease in metabolic power between the maximum and minimum values of the established ground truth curves. This accounts for an average reduction of $19.6 \pm 3.2 \%$. In comparison, the parameters found by the optimizer resulted in an average reduction of $18.0 \pm 4.6 \%$ in accordance to the established ground truth curves.

\section{Discussion \& CONCLUSION}

The experimental work presented in this paper shows that Body-in-the-Loop optimization can be a viable tool in the development and tuning of controllers for assistive robotic devices. For all subjects, the presented algorithm was able to autonomously minimize a physiological objective function in real-time. The key novelty in this context was that the optimization was based on physiological measurements of human effort. These are measurements that provided direct and objective feedback about the performance of the assistive device. From the perspective of a roboticist, this is a radically new way of human-robot-interaction. This interaction was neither based on a physical exchange between the robot and the user through forces and velocities (which are, however, used in the controller of the exoskeleton) nor was it based on the exchange of information through a designated user interface or a neuro-interface. Instead, the proposed methods allowed a robot to directly react to the physiological state of the user, a state which may not always be obvious or may not even be consciously known to the users themselves. With the development of more unobtrusive sensing technologies, such as skin conductance, heart rate, oxygen saturation, etc., this will allow a continuous and seamless adaptation of robots to their users. While the methods presented in this paper have focused on metabolic effort as the objective function, other physiological states might prove interesting candidates for optimization. This includes pain, comfort, training effort, and others. Of course, there is an even greater challenge of quantifying these states than of measuring metabolic effort.

Additionally, the proposed Body-in-the-Loop optimization techniques allow for parameter exploration of actuation shapes that may not be considered using traditional shaping methods. Currently, researchers shape actuation profiles of assistive devices to match actuation profiles of healthy, unassisted joints [21]. However, it has been shown that when using an adaptive controller, subjects may drive the controller adaptation to use abnormal actuation profiles that still appear to minimize a physiological objective [28]. Due to the inherent parameter exploration of optimization, Body-in-the-Loop optimization could potentially find optimal actuation shapes that may be disregarded by traditional shaping methods because they do not resemble healthy joint actuation profiles.

We fully acknowledge that at this stage, the presented concept of Body-in-the-Loop optimization is still limited. For one, the model we used to estimate metabolic effort combines any time constants associated with learning effects with the estimated time constant of the subject's metabolic system, $\tau$. For this initial study we felt that modeling learning effects would over complicate the methodology, so we attempted to wash out any learning effects by training subjects on the assistive devices for an adequate amount of time prior to data collection (day one of testing) [19]. Also, at this point the proposed sensor choice to estimate the physiological objective of metabolic effort from respiratory measurements is not practical for daily use outside of the lab setting. For Body-inthe-Loop optimization to be applicable for continuous use on assistive robotics, more discrete and transparent sensors and estimation methods need to be developed. Furthermore, we assessed the metabolic effort under deterministic conditions, being that subjects were walking with constant speed and on level ground. This ensured that changes in metabolic effort were strictly related to changes in the timing parameter. For the proposed methods to work outside the laboratory, we will have to extend the algorithms to control for any changes in the subjects locomotion pattern that might influence the physiological cost function. Such disturbances (such as varying walking speed or ground slope) must be either detected and compensated for, or data must be averaged over longer periods of time. Lastly, the devices used in this study are not an accurate representation of the current state of the art in ankle exoskeletons $[31,33]$. Our devices were tethered to an air supply which constrained them to only being used in a lab setting. Also, these exoskeletons were bulkier and heavier than the current state of the art, resulting in a larger increase in metabolic effort when comparing unpowered walking in the exoskeletons to walking with no exoskeletons at all. From pilot data, we saw that our exoskeleton design can reduce metabolic effort slightly below that of walking without the device when at an optimal parameter setting, yet this reduction was small. However, these exoskeletons were solely meant to perform as a platform to test the Body-in-the-Loop optimization techniques on and they greatly served that purpose.

Though we have validated that Body-in-the-Loop optimization is a viable option for the control of assistive robotic devices, we have only scratched the surface on the possible applications. We plan to expand upon the presented techniques to include alternative physiological objectives and sensing techniques to allow for discrete and continuous optimization during daily routines. Additionally, we plan to expand the techniques to include multidimensional optimization as most assistive devices can have upwards of ten shaping parameters [5]. This expansion into multidimensional space could have major implications to the field as the time required for brute force mapping of physiological objective functions grows exponentially with increased dimensionality. 


\section{REFERENCES}

[1] Hirotugu Akaike. A new look at the statistical model identification. Automatic Control, IEEE Transactions on, 19(6):716-723, 1974. URL http://dx.doi.org/10.1109/ TAC.1974.1100705.

[2] Samuel Au, Max Berniker, and Hugh Herr. Powered ankle-foot prosthesis to assist level-ground and stairdescent gaits. Neural Networks, 21(4):654-666, 2008. URL http://dx.doi.org/10.1016/j.neunet.2008.03.006.

[3] Jaehyun Bae, Maria De Rossi, Stefano Marco, Kathleen O’Donnell, Kathryn L Hendron, Louis N Awad, Thiago R Teles Dos Santos, Vanessa L De Araujo, Ye Ding, Kenneth G Holt, et al. A soft exosuit for patients with stroke: Feasibility study with a mobile off-board actuation unit. In Rehabilitation Robotics (ICORR), 2015 IEEE International Conference on, pages 131138. IEEE, 2015. URL http://dx.doi.org/10.1109/ICORR. 2015.7281188.

[4] Shalabh Bhatnagar, HL Prasad, and LA Prashanth. Stochastic recursive algorithms for optimization: simultaneous perturbation methods, volume 434. Springer, 2012.

[5] BiOM Personal Bionics. T2 product brochure. Accessed February 2015: http://www.biom.com/assets/2002129Rev-D-BiOM-T2-Product-Brochure-Web.pdf.

[6] Joaquin Blaya, Hugh Herr, et al. Adaptive control of a variable-impedance ankle-foot orthosis to assist dropfoot gait. Neural Systems and Rehabilitation Engineering, IEEE Transactions on, 12(1):24-31, 2004. URL http://dx.doi.org/10.1109/TNSRE.2003.823266.

[7] RL Blessey, HJ Hislop, RL Waters, and D Antonelli. Metabolic energy cost of unrestrained walking. Physical Therapy, 56(9):1019-1024, 1976.

[8] JM Brockway. Derivation of formulae used to calculate energy expenditure in man. Human Nutrition. Clinical Nutrition, 41(6):463-471, 1987.

[9] Kenneth P Burnham and David R Anderson. Kullbackleibler information as a basis for strong inference in ecological studies. Wildlife Research, 28(2):111-119, 2001.

[10] Kenneth P Burnham and David R Anderson. Model selection and multimodel inference: a practical information-theoretic approach. Springer Science \& Business Media, 2002.

[11] Stephen M Cain, Keith E Gordon, and Daniel P Ferris. Locomotor adaptation to a powered ankle-foot orthosis depends on control method. Journal of NeuroEngineering and Rehabilitation, 4:48, 2007. URL http://dx.doi. org/10.1186\%2F1743-0003-4-48.

[12] Joshua M Caputo and Steven H Collins. Prosthetic ankle push-off work reduces metabolic rate but not collision work in non-amputee walking. Scientific Reports, 4, 2014. URL http://dx.doi.org/10.1038/srep07213.

[13] Ching-Ping Chou and Blake Hannaford. Measurement and modeling of McKibben pneumatic artificial muscles.
Robotics and Automation, IEEE Transactions on, 12(1): 90-102, 1996. URL http://dx.doi.org/10.1109/70.481753.

[14] Steven H Collins, M Bruce Wiggin, and Gregory S Sawicki. Reducing the energy cost of human walking using an unpowered exoskeleton. Nature, 2015. URL http://dx.doi.org/10.1038/nature14288.

[15] Aaron M Dollar and Hugh Herr. Lower extremity exoskeletons and active orthoses: challenges and state-ofthe-art. Robotics, IEEE Transactions on, 24(1):144-158, 2008. URL http://dx.doi.org/10.1109/TRO.2008.915453.

[16] Dominic James Farris and Gregory S Sawicki. The mechanics and energetics of human walking and running: a joint level perspective. Journal of The Royal Society Interface, page rsif20110182, 2011. URL http://dx.doi. org/10.1098/rsif.2011.0182.

[17] Wyatt Felt, Jessica C Selinger, J Maxwell Donelan, and C David Remy. "Body-In-The-Loop": Optimizing Device Parameters Using Measures of Instantaneous Energetic Cost. PloS one, 10(8):e0135342, 2015. URL http://dx.doi.org/10.1371/journal.pone.0135342.

[18] Daniel P Ferris, Joseph M Czerniecki, and Blake Hannaford. An ankle-foot orthosis powered by artificial pneumatic muscles. Journal of Applied Biomechanics, 21(2):189, 2005. URL http://www.ncbi.nlm.nih.gov/pmc/ articles/PMC1351122/.

[19] S. Galle, P. Malcolm, W. Derave, and D. De Clercq. Adaptation to walking with an exoskeleton that assists ankle extension. Gait \& Posture, 38(3):495 - 499, 2013. URL http://dx.doi.org/10.1016/j.gaitpost.2013.01.029.

[20] Andrej Gams, Tadej Debevec, Tadej Petric, and Jan Babic. Metabolic cost of squatting using robotic knee exoskeleton. In Raad 2012 Proceeding. 21th International Workshop on Robotics in Alpe-Adria-Danube Region (Naples, 10-13 September 2012), page 184. ESA, 2012.

[21] Robert D Gregg and Jonathon W Sensinger. Towards biomimetic virtual constraint control of a powered prosthetic leg. Control Systems Technology, IEEE Transactions on, 22(1):246-254, 2014. URL http://dx.doi.org/10. 1109/TCST.2012.2236840.

[22] Abhishek Gupta, Marcia K O’Malley, Volkan Patoglu, and Charles Burgar. Design, control and performance of RiceWrist: a force feedback wrist exoskeleton for rehabilitation and training. The International Journal of Robotics Research, 27(2):233-251, 2008. URL http: //dx.doi.org/10.1177/0278364907084261.

[23] JP He, Rodger Kram, and Thomas A McMahon. Mechanics of running under simulated low gravity. Journal of Applied Physiology, 71(3):863-870, 1991. URL http://jap.physiology.org/content/71/3/863.

[24] Tibor Hortobágyi, Adria Finch, Stanislaw Solnik, Patrick Rider, and Paul DeVita. Association between muscle activation and metabolic cost of walking in young and old adults. The Journals of Gerontology Series A: Biological Sciences and Medical Sciences, 66(5):541-547, 2011. URL http:/dx.doi.org/10.1093/gerona/glr008. 
[25] Rachel W Jackson and Steven H Collins. An experimental comparison of the relative benefits of work and torque assistance in ankle exoskeletons. Journal of Applied Physiology, 119(5):541-557, 2015. URL http://jap.physiology.org/content/119/5/541.

[26] René Jimenez-Fabian and Olivier Verlinden. Review of control algorithms for robotic ankle systems in lowerlimb orthoses, prostheses, and exoskeletons. Medical Engineering \& Physics, 34(4):397-408, 2012. URL http://dx.doi.org/10.1016/j.medengphy.2011.11.018.

[27] Marcus W Kilpatrick, Robert R Kraemer, Edward J Quigley, Jennifer L Mears, Jeremy M Powers, Anthony J Dedea, and Nicholas F Ferrer. Heart rate and metabolic responses to moderate-intensity aerobic exercise: a comparison of graded walking and ungraded jogging at a constant perceived exertion. Journal of Sports Sciences, 27(5):509-516, 2009. URL http://dx.doi.org/10.1080/ 02640410802668650.

[28] Jeffrey R. Koller, Daniel A. Jacobs, Daniel P. Ferris, and C. David Remy. Learning to walk with an adaptive gain proportional myoelectric controller for a robotic ankle exoskeleton. Journal of NeuroEngineering and Rehabilitation, 12(1):1-14, 2015. URL http://dx.doi.org/ 10.1186/s12984-015-0086-5.

[29] Norman Lamarra, Brian J Whipp, Susan A Ward, and Karlman Wasserman. Effect of interbreath fluctuations on characterizing exercise gas exchange kinetics. Journal of Applied Physiology, 62(5):2003-2012, 1987.

[30] Paweł Maciejasz, Jörg Eschweiler, Kurt Gerlach-Hahn, Arne Jansen-Troy, and Steffen Leonhardt. A survey on robotic devices for upper limb rehabilitation. Journal of Neuroengineering and Rehabilitation, 11(3):10-1186, 2014. URL http://dx.doi.org/10.1186/1743-0003-11-3.

[31] Philippe Malcolm, Wim Derave, Samuel Galle, and Dirk De Clercq. A simple exoskeleton that assists plantarflexion can reduce the metabolic cost of human walking. PloS one, 8(2):e56137, 2013. URL http://dx.doi.org/10. 1371/journal.pone.0056137.

[32] Lynne V McFarland, Sandra L Hubbard Winkler, Allen W Heinemann, Melissa Jones, Alberto Esquenazi, et al. Unilateral upper-limb loss: satisfaction and prosthetic-device use in veterans and servicemembers from Vietnam and OIF/OEF conflicts. Journal of Rehabilitation Research \& Developement, 47(4):299-316, 2010. URL http://www.rehab.research.va.gov/jour/10/ 474/mcfarland.html.

[33] Luke M Mooney, Elliott J Rouse, and Hugh M Herr. Autonomous exoskeleton reduces metabolic cost of human walking during load carriage. Journal of NeuroEngineering and Rehabilitation, 11(5):80, 2014. URL http://dx.doi.org/10.1186/1743-0003-11-80.

[34] Jessica C. Selinger and J. Maxwell Donelan. Estimating instantaneous energetic cost during non-steadystate gait. Journal of Applied Physiology, 117(11):14061415, 2014. URL http://jap.physiology.org/content/117/ $11 / 1406$.
[35] Brian J Whipp. Rate constant for the kinetics of oxygen uptake during light exercise. Journal of Applied Physiology, 30(2):261-263, 1971. 